\title{
Pseudoaneurysm With A Fistula To The Right Ventricle Late After Surgical Repair of Type A Aortic Dissection in A Patient with Systemic Lupus Erythematosus: A Case Report
}

\author{
Akie Shimada \\ Juntendo University \\ Taira Yamamoto ( $\nabla$ tyamamo@juntendo.ac.jp ) \\ Juntendo University https://orcid.org/0000-0003-2214-2539 \\ Daisuke Endo \\ Juntendo University \\ Kousuke Nishida \\ Juntendo University \\ Satoshi Matsushita \\ Juntendo University \\ Tohru Asai \\ Juntendo University \\ Atsushi Amano \\ Juntendo University
}

\section{Research Article}

Keywords: Aortic dissection, systemic lupus erythematosus, Sjogren's syndrome, pseudoaneurysm, fistula, echocardiography-gated three-dimensional computed tomography

Posted Date: June 16th, 2021

DOI: https://doi.org/10.21203/rs.3.rs-556261/v1

License: (c) (i) This work is licensed under a Creative Commons Attribution 4.0 International License. Read Full License 


\section{Abstract}

Background: Pseudoaneurysm with a shunt to the right ventricle after aortic repair for acute aortic dissection is an extremely rare and life-threatening condition. Surgical treatment is unavoidable, but surgery is complicated, and there are some pitfalls. Herein, we explain the reoperation procedure performed for a patient at high surgical risk by clarifying the shunt site using multi-modality imaging before surgery.

Case presentation: A 69-year-old woman with a history of systemic lupus erythematosus (SLE) and Sjogren's syndrome presented with a pseudoaneurysm 1 year after emergency surgery for acute type A aortic dissection. Eight years after the first operation, she experienced sudden chest pain and presented to the emergency department. Her dyspnea also worsened; therefore, echocardiography and threedimensional computed tomography (3DCT) were performed, which revealed a pseudoaneurysm and a shunt to the right ventricle. The medical team attempted to close the shunt with a percutaneous catheter but was unsuccessful, and she was referred to our department for surgical treatment.

The pseudoaneurysm originating from the proximal side of the aorta was large with a diameter of 55 $\mathrm{mm}$, and echocardiography-gated 3DCT visualized the shunt from the pseudoaneurysm to the right ventricle. First, extracorporeal circulation was initiated, and a re-sternotomy was performed. We could not insert the left ventricular venting tube from the right side because of the size of the pseudoaneurysm; instead, the tube was inserted from the left atrial appendage. We found a half-circumferential disengaged anastomosis around the proximal anastomosis, which formed the large pseudoaneurysm leading to a fistula in the right ventricle. We closed the fistula and performed a Bentall operation.

The patient had a good postoperative course and was discharged on postoperative day 21. She continued treatment for SLE and Sjogren's syndrome and her inflammatory reaction improved.

Conclusion: We performed a Bentall operation and closure of a fistula with a re-sternotomy in a patient with type A aortic dissection with systemic lupus erythematosus and Sjogren's syndrome. Multimodal imaging is essential in defining the pseudoaneurysm and the fistula surrounding the anatomy while ensuring their resolution and guiding the approach for operation.

\section{Background}

Pseudoaneurysms after acute aortic dissection are reported to occur in $5-8 \%$ of cases [1], and the leading causes of this condition are severe calcification and fragility of the aortic wall, immature surgery, infection, and connective tissue diseases such as Marfan syndrome [2,3]. When disconnection occurs in the suture line between the artificial graft and the native aortic wall, there is an increased incidence of larger pseudoaneurysm development [4]. Furthermore, pseudoaneurysms can increase in size and extend directly into the right ventricle to form a fistula. This complication is infrequent, but cases of fistula formation in the right atrium, right ventricle, and pulmonary artery have been reported [5]. This condition 
is also critical in the control of acute heart failure because it causes rapid right heart overload hemodynamics.

Systemic lupus erythematosus (SLE) manifests as an inflammatory response in multiple organs, and the degree of inflammation reflects the severity and course of the disease. Recently, the effect of treatment with steroids and immunosuppressive drugs has increased the life expectancy of patients with SLE and revealed significant involvement of the cardiovascular system in the later stages of the disease [6]. Aortic dissection is not a common complication in patients with SLE, but its incidence is higher than that in ageand sex-matched controls [7]. Moreover, patients with SLE often have difficulties at the time of aortic dissection, including sudden back pain. However, it is not uncommon for aortic dissection to go undiagnosed until bilateral hemothorax or cardiac tamponade develops. [8].

Surgical repair is the primary treatment for pseudoaneurysms, but the mortality rate is high, and reoperation is often more dangerous than the initial surgery [9]. Furthermore, large pseudoaneurysms are challenging to approach surgically, and the thoracotomy itself becomes hazardous if it contacts the sternum, superior vena cava, right ventricle, or right atrium. Three-dimensional computed tomography (3DCT) with transesophageal echocardiography and electrocardiography can provide helpful information during preoperative planning.

Herein, we report the imaging and surgical procedures performed in a patient with a fistula in the right ventricle after acute aortic dissection with a history of SLE and Sjogren's syndrome.

\section{Case Presentation}

A 69-year-old woman presented with a chief complaint of exertional dyspnea. She experienced palpitations when climbing the stairs 3 months prior, and her dyspnea had since worsened. She had thrombocytopenic purpura at the age of 26 years and underwent a splenectomy. She was also diagnosed with SLE and Sjogren's syndrome at the age of 35 years and treated with steroids for 1 year for her SLE. The SLE was well controlled after the end of treatment. She had been on medical therapy for high blood pressure (olmesartan medoxomil, $10 \mathrm{mg}$ per day) and underwent endoscopic treatment for stomach cancer. She had no history of smoking. Ten years prior, she was diagnosed with acute aortic dissection (Stanford type A), and hemiarch aortic replacement was performed with an artificial graft. She was noted to have a small pseudoaneurysm at the proximal anastomosis 1 year postoperatively. She had also experienced sudden-onset dyspnea and chest pain 1 year prior to the current presentation. She was rushed to the hospital where she had undergone her first surgery and was treated for acute heart failure. Contrast-enhanced CT revealed a pseudoaneurysm perforating the right ventricle, and surgical treatment was considered necessary. The reoperation was deemed technically challenging and a high surgical risk because the pseudoaneurysm was in contact with the sternum. Catheter-based fistula closure was planned because of the high risk, but could not be performed in the first hospital. She requested surgery at our hospital and was transferred. 
Physical examination revealed a height of $160.6 \mathrm{~cm}$ and weight of $48.1 \mathrm{~kg}$. Her blood pressure was 110/56 $\mathrm{mmHg}$, and her heart rate was $66 \mathrm{bpm}$ in sinus rhythm. Her oral medications were furosemide 40 $\mathrm{mg} /$ day, tolvaptan $15 \mathrm{mg} /$ day, and bisoprolol fumarate $5 \mathrm{mg} /$ day. The laboratory data were as follows: hemoglobin, $11.2 \mathrm{~g} / \mathrm{dL}$; platelet count, $196 \times 109 \mathrm{U} / \mathrm{L}$; total protein, $7.5 \mathrm{mg} / \mathrm{dL}$; albumin, $3.9 \mathrm{mg} / \mathrm{dL}$; triglyceride, $90 \mathrm{mg} / \mathrm{dL}$; low-density lipoprotein cholesterol, $81 \mathrm{mg} / \mathrm{dL}$; high-density lipoprotein cholesterol, $75 \mathrm{mg} / \mathrm{dL}$; serum-creatinine, $1.00 \mathrm{mg} / \mathrm{dL}$; hemoglobin A1c (HbA1c), 5.7\%; and brain natriuretic peptide (BNP), 718 pg/; C-reactive protein, 0.15 mg/L; fibrin degradation product (FDP) D-dimer, 3.2 $\mu \mathrm{g} / \mathrm{ml}$; antinuclear antibody positive; anti-Smith antibody negative; double-stranded DNA IgG 15 IU/mL; antiphospholipid (antibody) syndrome negative; anti SS-A/SS-B antibody negative; antiphospholipid antibodies negative; and antineutrophil cytoplasmic antibody (PR3/MPO) 1.0/1.0 U/mL.

Electrocardiography revealed normal sinus rhythm. Chest radiography revealed a cardiothoracic ratio of $60 \%$ (Fig. 1), the diameter of the proximal arch was $45 \mathrm{~mm}$ on $\mathrm{CT}$, the pseudoaneurysm of the proximal anastomosis was $60 \mathrm{~mm}$, and Valsalva was $37 \mathrm{~mm}$ (Fig. 2). The diameter of the annulus was $21 \mathrm{~mm}$ with mild to moderate aortic valve regurgitation, the shunt flow into the right ventricle from this pseudoaneurysm was recognized, and the pulmonary blood flow/systemic blood flow ratio (Qp/Qs) was 1.05 (Fig. 3). Her left ventricular ejection fraction was $68 \%$, with mild tricuspid regurgitation, and the estimated right ventricular pressure was 39 mmHg (risk score: Euro II 20.25\%, Japan Score 10.9\%). Electrocardiography-gated cardiac 3DCT was performed preoperatively; the pseudoaneurysm was located in the proximal anastomosis, and the morphology of the Valsalva was preserved. The shunt perforated the right ventricle and was not in contact with the right coronary artery. We found it challenging to dissect the pseudoaneurysm before establishing cardiopulmonary bypass (CPB) in the re-aortic surgery.

A sternotomy was performed in a similar manner as the previous surgery. Before adhesion detachment, an artificial CPB was established with the inflow on the right subclavian artery and the outflow on the right femoral vein and was placed into the left atrial venting tube via the left atrial appendage. We exfoliated the tissue surrounding the pseudoaneurysm and placed a tape around the distal superior vena cava. We performed extended graft replacement by removing the previous graft under systemic hypothermia with selective cerebral perfusion. Regarding the strategy for brain protection, the target minimum core temperature was approximately $28^{\circ} \mathrm{C}$. After distal anastomosis, systemic rewarming was initiated. At the ST junction, the artificial graft and aortic wall were separated by half of the posterior wall (Fig. 4A). We were able to identify a fistula leading from the pseudoaneurysm to the right ventricle (Fig. 4B), which was closed while reinforcing the fistula with a pericardial patch. Because the Valsalva wall was fragile and vulnerable, aortic root reconstruction was performed using the bio-Bentall technique (Inspiris 23 mm: Edwards Lifesciences, Irvine, CA, USA; Gelweave Valsalva 26 mm: Vascutek Ltd., Terumo Aortic, Scotland, UK). The pathological findings of the pseudoaneurysm showed that the aortic intima and media were absent, and only the adventitia was preserved. The operative, CPB, aortic cross-clamp, and circulatory arrest times were 466, 252, 189, and 25 min, respectively. The respiratory support time was $12 \mathrm{~h}$ postoperatively. She was discharged from the intensive care unit on postoperative day 2 , and rehabilitation was initiated. She experienced postoperative atrial fibrillation; however, no other complications occurred. The hemodynamic performance was excellent without a shunt and there was no 
evidence of pseudoaneurysm on postoperative 3DCT (Fig. 5). One year after the operation, the patient was able to effectively resume daily activities, and we found no residual shunt on postoperative echocardiography.

\section{Discussion And Conclusions}

Postoperative pseudoaneurysms of the ascending aorta are associated with periportal extension and high mortality [10]. The actual incidence of pseudoaneurysm formation after cardiac surgery is unknown but is estimated to be $<0.5 \%$ [11]. As the pseudoaneurysm expands, compression of the surrounding tissue structures, infection, rupture or perforation, and a sudden hemodynamic collapse that can turn fatal may occur. The only treatment is surgical repair, but the mortality rate is reportedly as high as $15.4 \%$. Recently, the percutaneous approach has been applied with increasing frequency, particularly in patients at high surgical risk [12].

It is easy to identify a pseudoaneurysm in the postoperative period using imaging studies. However, in cases of sudden onset of heart failure, an immediate diagnosis is required, as in the present case. Transesophageal echocardiography can clearly indicate the origin of the pseudoaneurysm and whether the fistula is penetrating but does not allow for the determination of the surgical approach for reoperation. Contrast-enhanced CT provides a better understanding of the pseudoaneurysm location and its relationship to the sternum and surrounding aorta, superior aorta, pulmonary artery, and pulmonary vein, and is an effective tool for determining the surgical approach for reoperation. However, when a pseudoaneurysm forms a shunt in the right ventricle, it is challenging to determine the exact positional relationship between the aortic root and the right coronary artery using conventional imaging studies. Echocardiography-gated 3DCT clearly depicts the aortic root and its positional relationship with the coronary origin and right coronary artery, and we believe that these factors are essential for preoperative planning. Multimodal imaging is important for revealing the pseudoaneurysm and surrounding anatomy, guiding treatment, and ensuring surgical success [13].

It has been reported that four major challenges must be overcome in such surgery: 1) rupture of the pseudoaneurysm during the reopening of the chest, 2) cerebral perfusion during circulatory arrest, 3 ) ventricular distension due to aortic valve insufficiency, and 4) increased right and left shunt volume during CPB [14].

In cases in which the sternum is in close contact with the pseudoaneurysm, the sternal incision itself may cause a fatal rupture, and it is imperative to establish CPB prior to the sternal incision.

In the presence of aortic insufficiency or a shunt from a pseudoaneurysm, progressive myocardial protection cannot be accurately injected, and retrograde myocardial protection is adequate. In addition, ventricular fibrillation occurs during the transition to hypothermia, and this condition requires good left ventricular venting because the left ventricle expands rapidly. Further, circulatory arrest by simple hypothermic perfusion should be avoided in aortic regurgitation because of the high likelihood of hypothermia-induced ventricular fibrillation and subsequent ventricular filling. 
If there is a large pseudoaneurysm on the right side of the longitudinal wall, the right pulmonary vein cannot be identified, and the right atrium cannot be incised unless the superior vena cava can be identified. The venting tube may be placed via the left atrial appendage in this situation. If the adhesions are strict to the chest wall, a left ventricular vent may be inserted through the transthoracic wall $[15,16]$. Intraoperative transesophageal ultrasonography is the most effective method for observing this condition. In this situation, information obtained from intraoperative transesophageal ultrasound can be beneficial.

An increased relative risk of developing aneurysmal lesions has been reported in patients diagnosed with $\operatorname{SLE}[7,17]$. An increased relative risk has also been reported for aortic dissection, with older age, male sex, SLE duration of $>3$ years, and hypertension reported as risk factors for both aortic aneurysm and dissection [7]. In terms of pathological findings, histological findings of diffuse aortitis are noted in cases of aortic dissection in patients with SLE, and medial cystic changes and loss of elastic fibers are recognized as the primary lesions $[18,19]$. In the present case, we believe that SLE had little effect on the patient because the inflammatory response was negative and stable after 1 year of steroid treatment after the diagnosis of SLE. However, the pathological findings included a marked lack of intima and tunica media, and we must consider that inflammation of the aorta may have existed at the time of surgery.

The limitation of this report is that there was no previous medical history, and the association with SLE or Sjogren's syndrome is not clear. In this case, the patient abruptly developed a shunt and heart failure, but we could not determine whether the patient would have stabilized if we waited or whether emergency surgery would have been necessary and desirable. In addition, because of the failure of the first catheterization, we opted for surgical treatment from the beginning and did not attempt catheterization.

In conclusion, we performed a Bentall operation and closure of the fistula with resternotomy in a patient with type A aortic dissection and with SLE and Sjogren's syndrome. A multidisciplinary approach, including a medical doctor's diagnosis, is essential for accurate surgery.

\section{List Of Abbreviations}

Systemic lupus erythematosus (SLE), three-dimensional computed tomography (3DCT), hemoglobin A1c (HbA1c), brain natriuretic peptide (BNP), fibrin degradation product (FDP), cardiopulmonary bypass (CPB)

\section{Declarations}

Patient's perspectives: The patient in the presented case was satisfied with explanations of their treatment before surgery and the postoperative course and test results

\section{Ethics approval and consent to participate}


Written consent was obtained from the patient for publication of the case report. The Clinical Ethics Committee of Juntendo University Hospital approved this study (JHS21-003), and the study was conducted in accordance with the principles of the Helsinki Declaration.

\section{Consent for publication}

Written consent was obtained from the patient regarding the publication of their personal information.

\section{Availability of data and materials}

Data associated with this manuscript are not publicly available but can be made available by the corresponding author upon reasonable request.

\section{Competing interests}

The authors declare that they have no competing interests.

\section{Funding}

Not applicable.

\section{Authors' contributions}

AS collected and analyzed the patient's data and was a major contributor to the writing of the manuscript. TY was the primary operator in this case, organized the team for these surgical and medical treatments, and analyzed the patient's data. DE, KN SM, and TA took care of postoperative management and collected the data for this patient. AA supervised the work. All authors have read and approved the final manuscript.

\section{Acknowledgements}

We would like to thank Editage (www.editage.com) for English language editing.

\section{References}

1. Luciani N, De Geest R, Lauria G, Farina P, Luciani M, Glieca F, et al. Late reoperations after acute aortic dissection repair: single-center experience. Asian Cardiovasc Thorac Ann. 2015;23:787-94.

2. Concistrè G, Casali G, Santaniello E, Montalto A, Fiorani B, Dell'Aquila A, et al. Reoperation after surgical correction of acute type A aortic dissection: risk factor analysis. Ann Thorac Surg. 2012;93:450-5.

3. Tanaka H, Okada K, Kawanishi Y, Matsumori M, Okita Y. Clinical significance of anastomotic leak in ascending aortic replacement for acute aortic dissection. Interact Cardiovasc Thorac Surg. 2009;9:209-12. 
4. Malvindi PG, van Putte BP, Sonker U, Heijmen RH, Schepens MA, Morshuis WJ. Reoperation after acute type A aortic dissection repair: a series of 104 patients. Ann Thorac Surg. 2013;95:922-7.

5. Spier LN, Hall MH, Nelson RL, Parnell VA, Pogo GJ, Tortolani AJ. Aortic dissection: rupture into right ventricle and right pulmonary artery. Ann Thorac Surg. 1995;59:1017-9.

6. Svenungsson E, Jensen-Urstad K, Heimbürger M, Silveira A, Hamsten A, de Faire U, et al. Risk factors for cardiovascular disease in systemic lupus erythematosus. Circulation. 2001;104:1887-93.

7. Wang SH, Chang YS, Liu CJ, Lai CC, Chen TJ, Chen WS. Incidence and risk analysis of aortic aneurysm and aortic dissection among patients with systemic lupus erythematosus: a nationwide population-based study in Taiwan. Lupus. 2014;23:665-71.

8. Wei HY, Chung HT, Wu CT, Huang JL. Aortic dissection complicated with hemothorax in an adolescent patient with systemic lupus erythematosus: case report and review of literature. Semin Arthritis Rheum. 2011;41:12-8.

9. Geirsson A, Bavaria JE, Swarr D, Keane MG, Woo YJ, Szeto WY, et al. Fate of the residual distal and proximal aorta after acute type a dissection repair using a contemporary surgical reconstruction algorithm. Ann Thorac Surg. 2007;84:1955-64. discussion 1955.

10. El Oumeiri B, Louagie Y, Buche M. Reoperation for ascending aorta false aneurysm using deep hypothermia and circulatory arrest. Interact Cardiovasc Thorac Surg. 2011;12:605-8.

11. Atik FA, Navia JL, Svensson LG, Vega PR, Feng J, Brizzio ME, et al. Surgical treatment of pseudoaneurysm of the thoracic aorta. J Thorac Cardiovasc Surg. 2006;132:379-85.

12. Preventza O, Henry MJ, Cheong BY, Coselli JS. Endovascular repair of the ascending aorta: when and how to implement the current technology. Ann Thorac Surg 108. 2014;97:1555-60.

13. Schwartz J, Burstein B, Kovacina B, Martucci G, Abualsaud A, Afilalo J, et al. Percutaneous closure of a giant aortic pseudoaneurysm using multimodality imaging guidance. Can $\mathrm{J}$ Cardiol. 2021;30:0828-282X. doi:10.1016/j.cjca.2021.01.019. :(21)00064 - 7.

14. Reents W, Kenn W, Babin-Ebell J, Leyh RG, Gorski A. False aneurysm with aortopulmonary shunt after replacement of the ascending aorta. J Thorac Cardiovasc Surg. 2010;139:e62-3.

15. Wakefield BJ, Leone AJ, Sale S. Left ventricular apex venting in high-risk redo sternotomy with severe aortic insufficiency: a case report. A A Pract. 2018;10:16-9.

16. Doig F, Naidoo R, Sharma V, Tesar P. A unique hybrid approach to complex re-do aortic root surgery. Heart Lung Circ. 2017;26:e68-70. doi:10.1016/j.hlc.2017.05.126.

17. Guy A, Tiosano S, Comaneshter D, Tekes-Manova D, Shovman O, Cohen AD, et al. Aortic aneurysm association with SLE - a case-control study. Lupus. 2016;25:959-63.

18. Brinster DR, Grizzard JD, Dash A. Lupus aortitis leading to aneurysmal dilatation in the aortic root and ascending aorta. Heart Surg Forum. 2009;12:E105-8.

19. Silvestri V, Simonte G. Aortic pathology in systemic lupus erythematosus: A case report and review of literature. Ann Vasc Surg. 2017;43:312:e5. 312.e12. doi: 10.1016/j.avsg. 
Figures

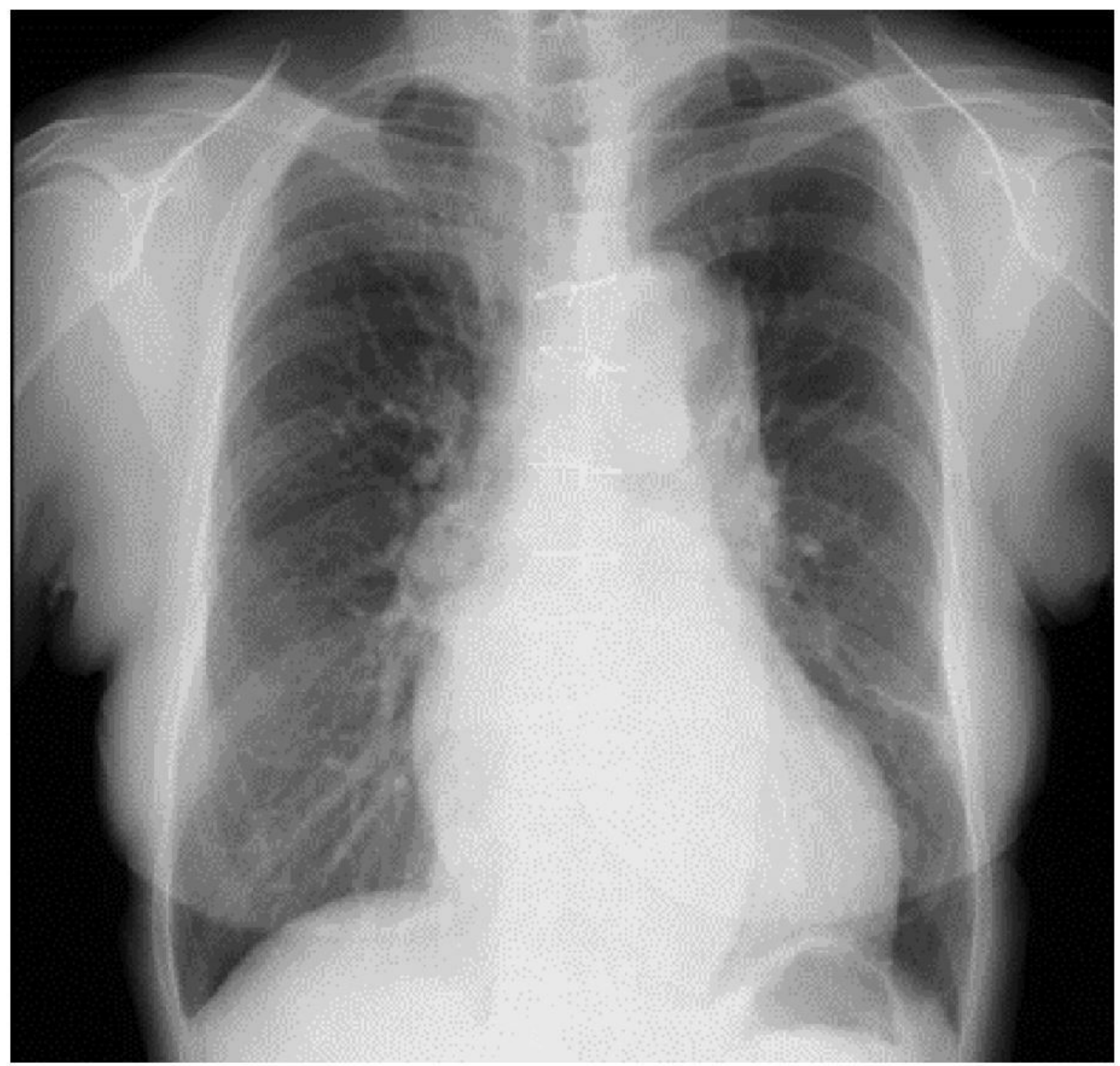

Figure 1

Preoperative chest $\mathrm{X}$-rays 
a

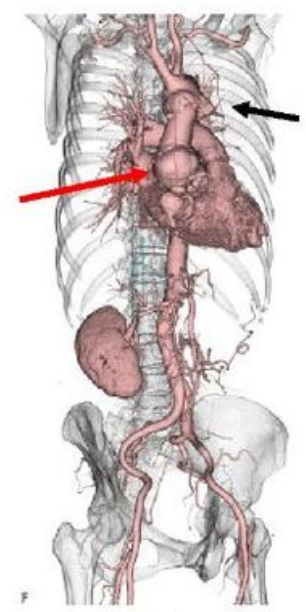

d

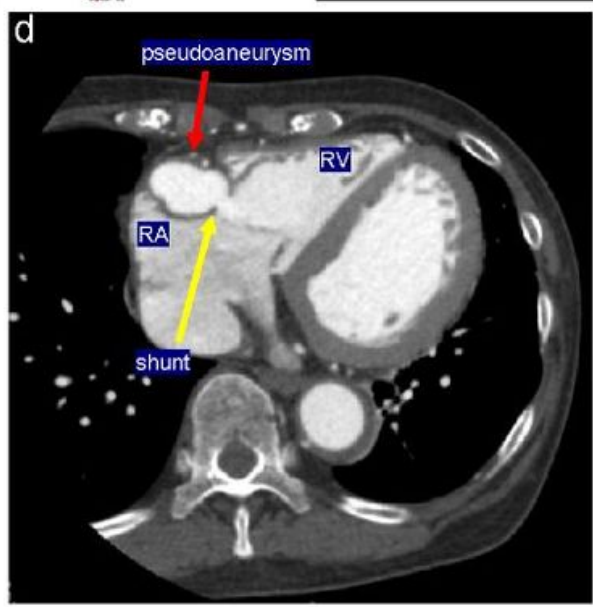

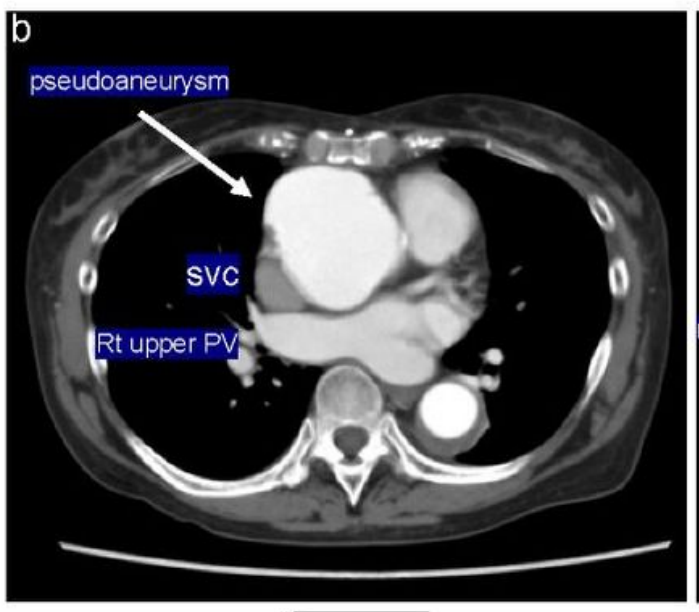
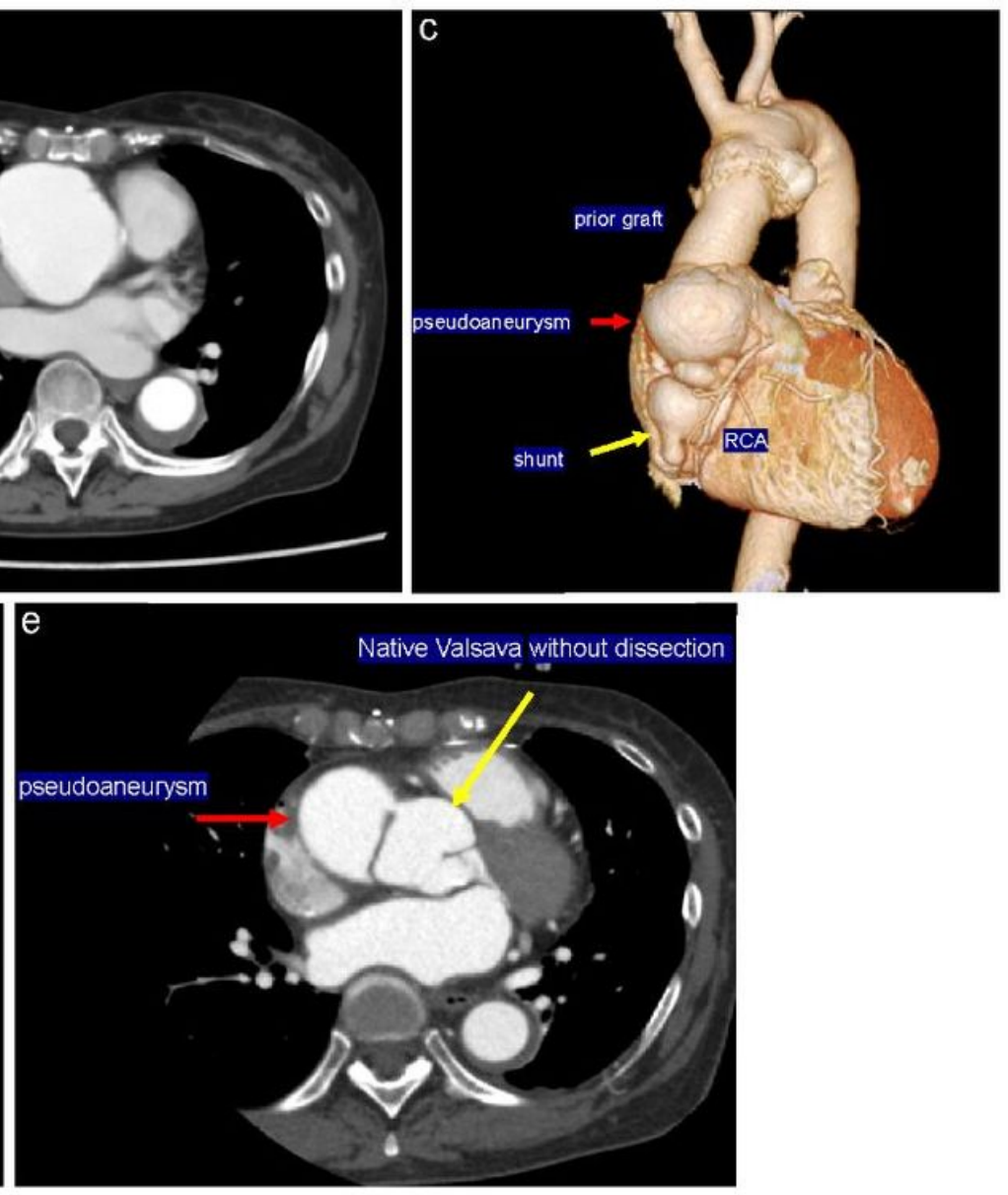

Figure 2

Preoperative 3DCT images (A) Preoperative 3DCT showing the distal (black arrow) and distal (red arrow) pseudoaneurysm. (B) The pseudoaneurysm (white arrow) is in close contact with the sternum. (C) ECGgated 3DCT is used to visualize the pseudoaneurysm (red arrow) and the previously used artificial graft ("prior graft"). The pseudoaneurysm is not in direct contact with the right coronary artery, and there is a small distance between them. (D) The pseudoaneurysm (red arrow) and the shunt (yellow arrow) are shown near the right heart structures. (E) The Valsalva without the aortic dissection (yellow arrow) is shown with the pseudoaneurysm (red arrow). 3DCT, three-dimensional computed tomography; ECG, echocardiography; SVC, superior vena cava; rt upper PV, right upper pulmonary vein; RA; right atrium. RV; right ventricle; RCA, right coronary artery. 


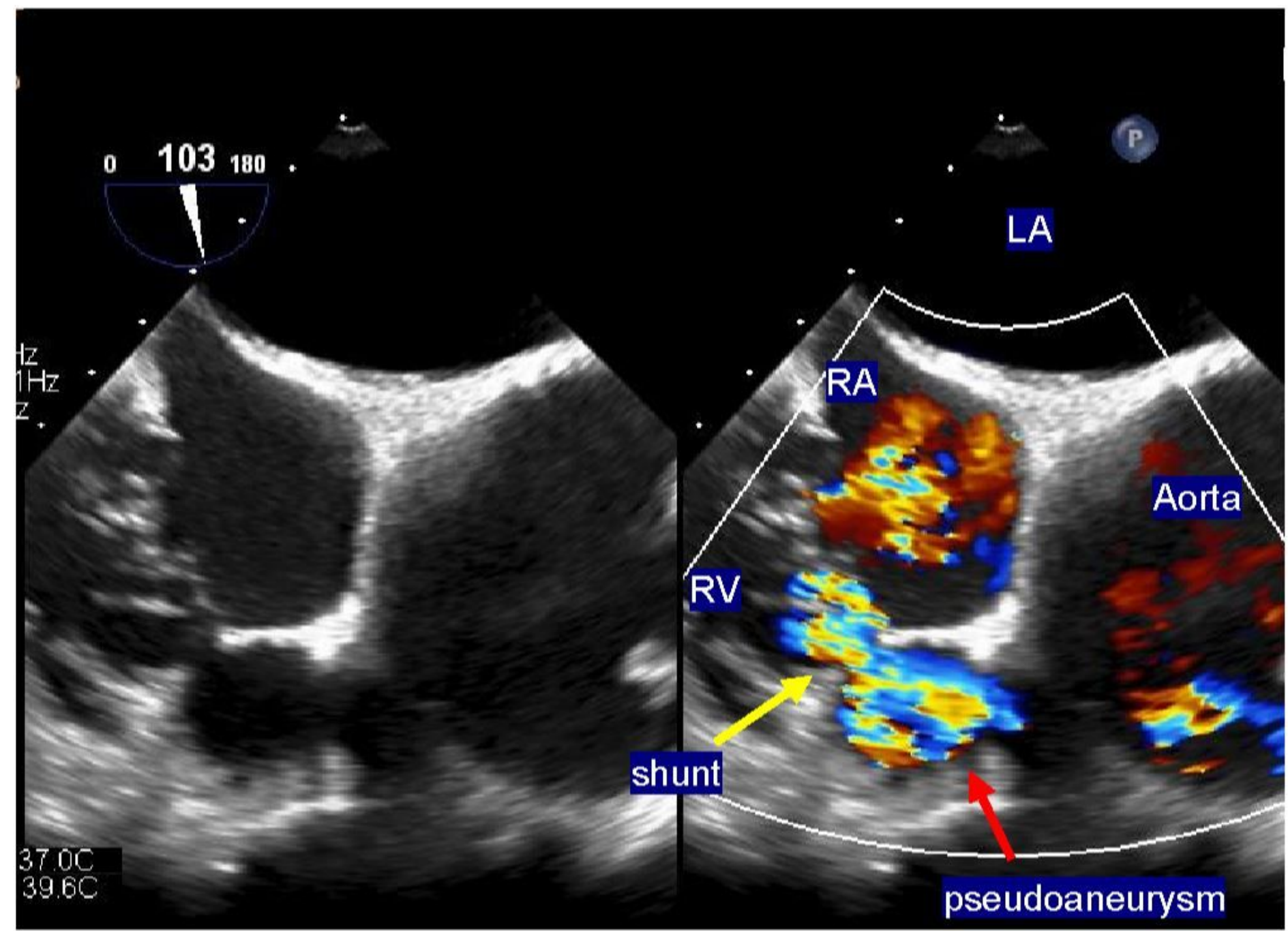

\section{Figure 3}

Transesophageal cardiac ultrasonography The pseudoaneurysm (red arrow) is shown on transesophageal cardiac ultrasonography shunt (yellow arrow) are also indicated. RA, right atrium; RV, right ventricle; LA, left atrium. 

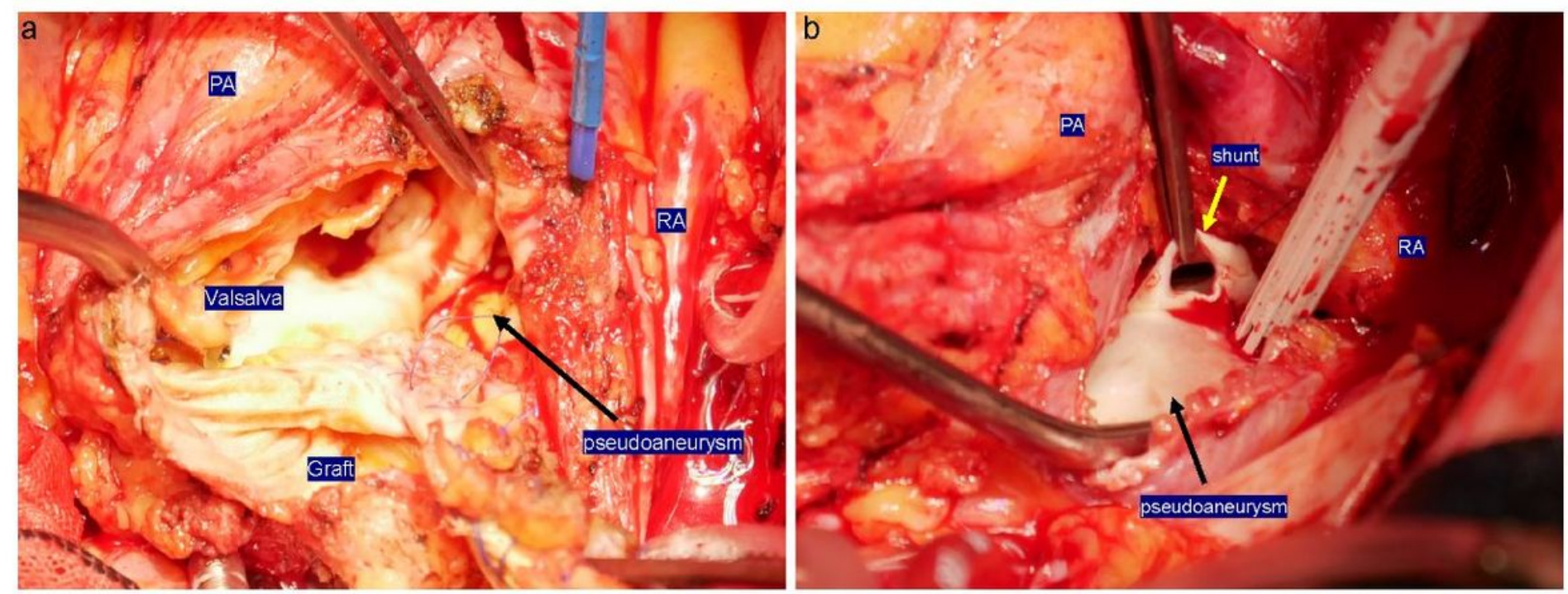

\section{Figure 4}

Operative findings (A) Intraoperative view showing the pseudoaneurysm (black arrow). At the ST-junction, the artificial graft and the aortic wall are separated by half of the posterior wall. (B) Intraoperative view showing the pseudoaneurysm's (black arrow) proximity to the shunt (yellow arrow). RA, right atrium; LA, left atrium 


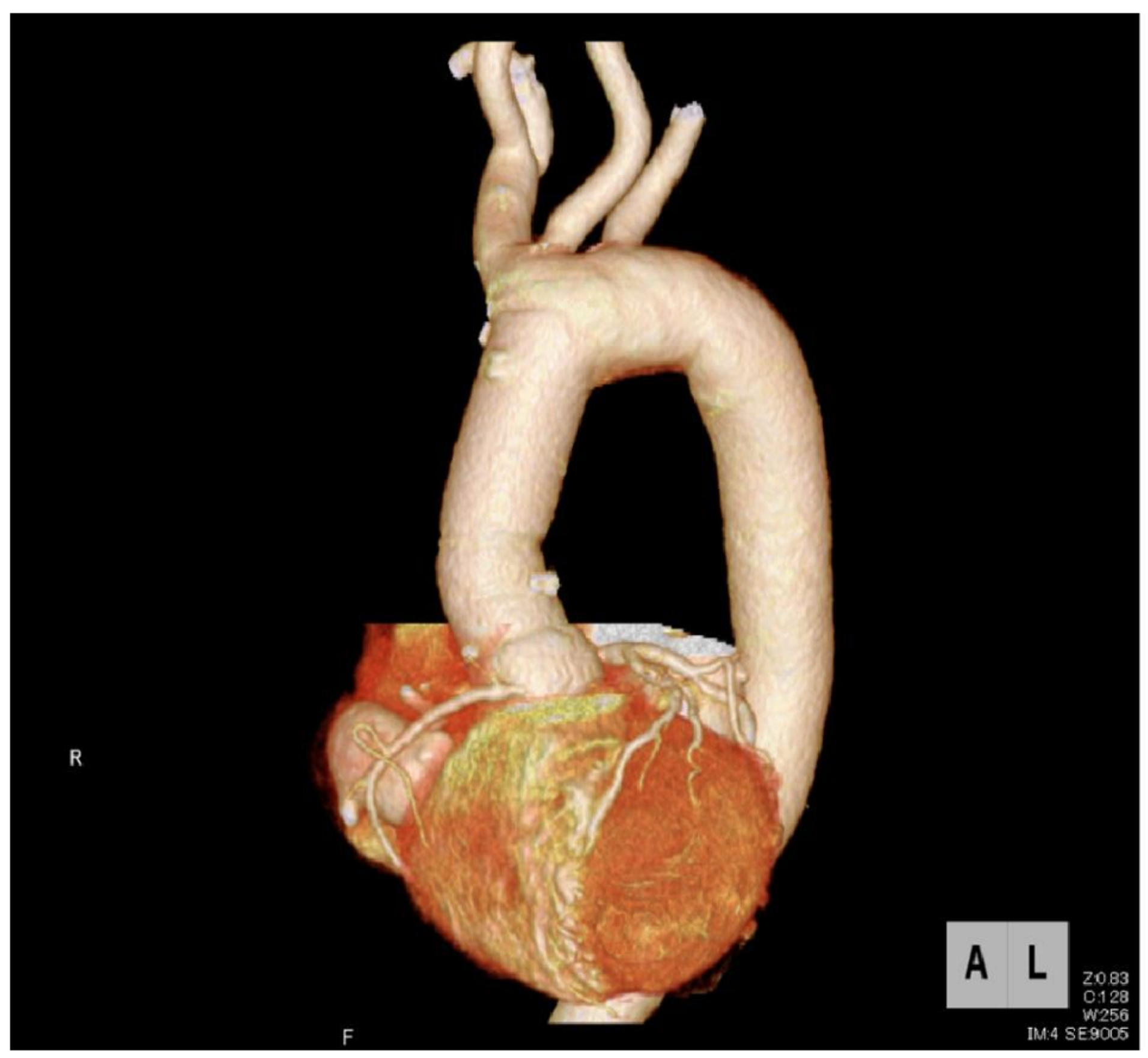

Figure 5

Postoperative ECG-gated 3DCT 3DCT showing no pseudoaneurysm and a normal right coronary artery. ECG, echocardiography; 3DCT, three-dimensional computed tomography;

\section{Supplementary Files}

This is a list of supplementary files associated with this preprint. Click to download.

- CAREchecklistEnglish2013Shimada.pdf 